\title{
Moving daily average of the hourly magnetic field values - the example of usage at Novosibirsk Observatory during 2011 (results and prospects)
}

\author{
Nikolay Semakov ${ }^{1,2}$, Aleksandr Kovalev $^{1}$, Anatoliy Pavlov ${ }^{1,2}$, Olga Fedotova $^{1}$ \\ ${ }^{1}$ Institute of Petroleum Geology and Geophysics SB RAS, Russia \\ ${ }^{2}$ Novosibirsk State University, Russia
}

\begin{abstract}
The parameters of the equivalent central dipole calculated using hourly values of the magnetic field elements during 2011: the angular elements transformed to the hourly values of the geographic coordinates of the North magnetic pole and the intensity values transformed to local magnetic constant. Next step is the calculation of the daily mean values at every hour. This method applied to both current digital data and historical data presented as monthly tables of hourly values. The advantage of method is its ability to show the changes of the magnetic field independently from daily variation. Using of the "integrate" parameters (the magnetic pole coordinates and local magneto constant) allows detect the regional features of its variations. The features in the daily values compared with anomalous geological and geophysical events observed in the past and predicted in the near future.
\end{abstract}

\section{Introduction}

The issue of geomagnetic precursors of earthquakes has a rich history and strong justifications for their detection and analysis. The Earth's magnetic field is able to respond to varying degrees of change in the tense state of the Earth's crust associated with the preparation of events such as volcanic eruptions and strong earthquakes, sometimes causing great trouble.

One of the largest earthquakes, accompanied by a devastating tsunami, was the Great Earthquake of Eastern Japan on March 11, 2011. This event is dedicated to many publications, affecting, among others, and features of the behavior of the magnetic field. On the example of this earthquake, the authors of the article, employees of the Magnetic Observatory "Novosibirsk", hope to draw the attention of geophysicists to the possibility of applying a new approach to the detection of geomagnetic harbingers of such unique phenomena.

\footnotetext{
${ }^{*}$ Corresponding author: semakovnn@ipgg.sbras.ru
} 


\section{"Power" and angular characteristics of the magnetic field}

The vast majority of the works devoted to the theme considered variations of one, less often two or more "powerful" elements of terrestrial magnetism. There are five of these elements: the magnetic induction vector module $(\mathrm{F})$, as well as its vertical $(\mathrm{Z})$, horizontal $(\mathrm{H})$, northern $(\mathrm{X})$ and eastern $(\mathrm{Y})$ components. In addition, there are two other angular elements: magnetic declination (D) magnetic inclination (I), which analyzed as possible harbingers much less frequently.

Magnetic declination is the angle between the geographic and magnetic meridian at a given point (positive values - east of the geographical meridian). The north arrow of the compass shows the direction to the northern virtual magnetic pole. Magnetic inclination is the angle between the horizontal plane and the magnetic induction vector (positive values are downward, as well as for the Z). The angle of inclination determines the distance from the observation point to the magnetic pole in the arc of a large circle. It is possible to convert these four values into the geographical coordinates of any of the magnetic poles equivalent to the central dipole, knowing the angles of declination and inclination, as well as the geographical coordinates of the observation point. Everybody magnitologist have long been accustomed to calling such calculated poles virtual. The north virtual magnetic pole is the point of "exit" to the earth's surface of the southern "end" of the dipole, located in the center of the globe, and the opposite of it virtual magnetic pole shows the place of "exit" to the surface of the northern "end" of this central dipole.

Digital registration of $\mathrm{X}, \mathrm{Y}$, and $\mathrm{F}$ is carried out continuously in most modern magnetic observatories, including the observatories that make up the INTERMAGNET (INTErnational Real MAGnetic NET )[1]. An example of such a record in the form of a magnitograms shown in Figure 1.

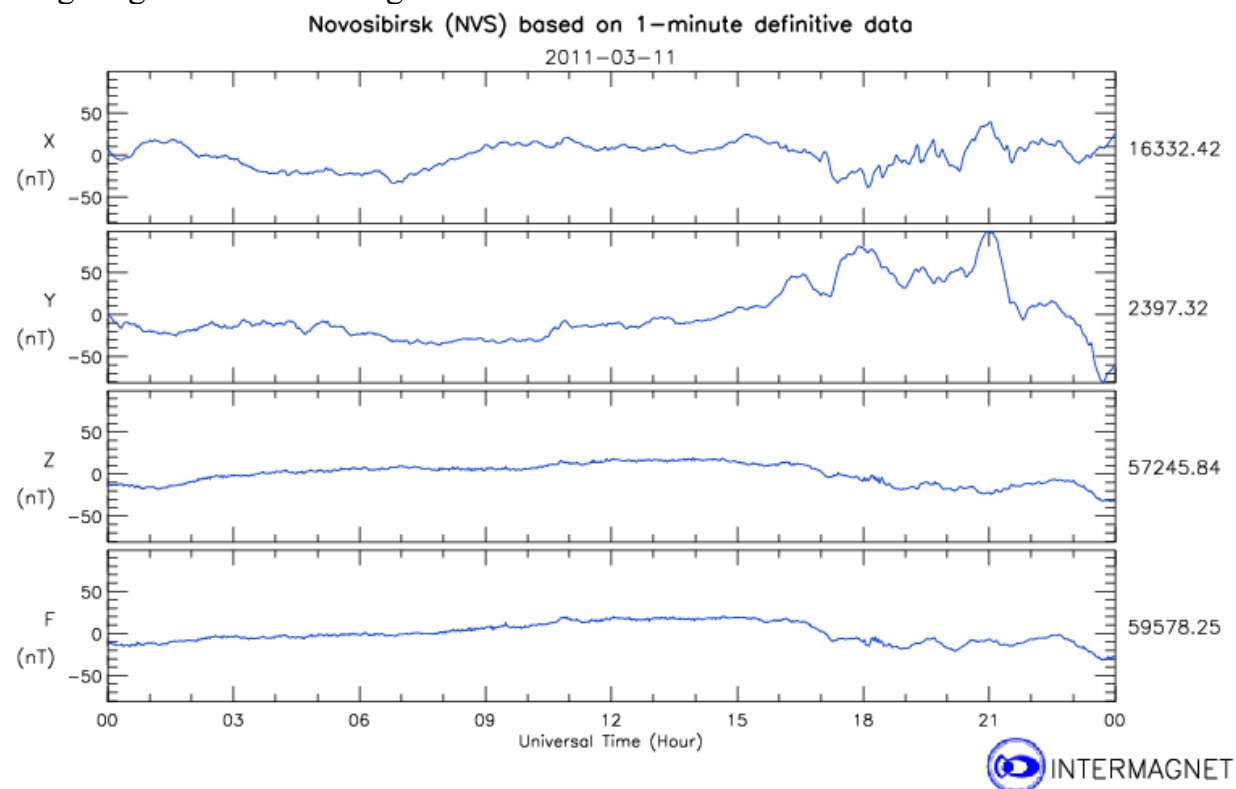

Fig 1. "Power" elements of terrestrial magnetism for 11.03.2011 at the Novosibirsk Observatory. 
The above "powerful" elements of terrestrial magnetism would be more correctly called "power-corner" because the value measured at that point of each of them changes not only when the magnetic moment of the equivalent dipole changes, but also when its orientation changes. Note that magnetic declination and inclination remain constant when the magnetic moment of the equivalent central dipole changes, but its orientation does not change.

Our approach to geomagnetic harbingers has two characteristics. The first is to use purely forceful and purely angular characteristics of the magnetic field. Their separation makes it possible to separate and consider separately the features of geomagnetic variations associated with the change in the magnetic moment of the equivalent central dipole and with the change in its orientation. This approach is particularly appropriate in the study of temporal variations, since changing the magnetic moment of the equivalent magnetic dipole and changing its orientation over time may have a mutually exclusive effect on each of the five so-called "force" elements of terrestrial magnetism measured at this point. For example, the module of the magnetic induction vector in the Novosibirsk Observatory has been gradually increasing in recent years (in February 2020, the average daily values of $\mathrm{F}$ exceeded $60,000 \mathrm{~nL}$ ). At the same time, the magnetic moment of the equivalent central dipole according to this observatory decreases. A similar pattern observed in certain periods and in other regions of the globe.

The second feature is the use of "integral" characteristics. Such an integral force characteristic of the magnetic field at this point are the local magnetic constant, and the integral angular characteristic - the coordinates of the northern virtual magnetic pole.

Local magnetic constant $(\mathrm{G})$ is extremely rarely used to analyze the observed magnetic field. Therefore, it is necessary to say a few words in the "defense" of this remarkable characteristic with the hope of more active use of it by fellows in the future. Calculations of local magnetic constant make by L.A. Bauer more than 100 years ago to compare the magnitude of the Earth's magnetic moment by observations in different regions [2]. Value of $\mathrm{G}$ associate with the magnetic moment of the equivalent dipole (M) and the distance $(\mathrm{R})$ from the observation point to this dipole a simple ratio (1):

$$
\mathrm{M}=\mathrm{G}^{*} \mathrm{R}^{\wedge} 3
$$

Note that under the equivalent dipole in this formula can be considered not only dipole central (i.e. located in the center of the globe) or radial (i.e. shifted by the radius of the Earth, but directed along of this radius), but also any displaced dipole. However, to estimate the degree of dipolarity of the magnetic field, as well as for comparing other characteristics of the magnetic field by the observations in different regions, it is most convenient to "place" the equivalent dipole in the center of the globe. This is also important because only for the central and radial dipoles it makes sense to calculate the position of magnetic poles in their usual understanding: as points on the Earth's surface, in which the freely suspended magnetic arrow positioned vertically. "Dipolarity" is "the ability to present the analyzed magnetic field as a single magnetic dipole"[3]. It is clear that, with this understanding, dypolarity can be global, regional and even local. To calculate the $\mathrm{G}$ value by the elements of terrestrial magnetism measured at a given point, you can use a formula (2) or (3): 


$$
\begin{aligned}
& \mathrm{G}=\left(\mathrm{H}^{\wedge} 2+0.25 \mathrm{Z}^{\wedge} 2\right)^{\wedge} 0.5 \\
& \mathrm{G}=\mathrm{F}^{*}\left(1-0.75(\sin \mathrm{I})^{\wedge} 2\right)^{\wedge} 0.5
\end{aligned}
$$

A distinctive feature of the $\mathrm{G}$ is the fact that it remains constant at any orientation of the magnetic dipole, if the distance from the point of observation to this dipole is constant and the magnetic moment of the dipole does not change. In particular, if the Earth was a ball and had a single randomly oriented central dipole, at any point on the surface of this ball the value of $\mathrm{G}$ would be the same (currently about 30,000 nT). It is this "sameness" of the local magnetic constant "guilty" in its name, which to some may seem not quite successful. It is also important to note that in order to calculate the magnitude of G there is no need to measure magnetic declination at a given point, i.e. to produce in addition to magnetic and astronomical observations.

\section{Features of local magnetic constant behavior}

Figure 2 shows the average hourly values of the local magnetic constant for the Novosibirsk Magnetic Observatory for the first 4 months of 2011. The smoother curve in this chart reflects the progress of the moving daily average with one hour increment.

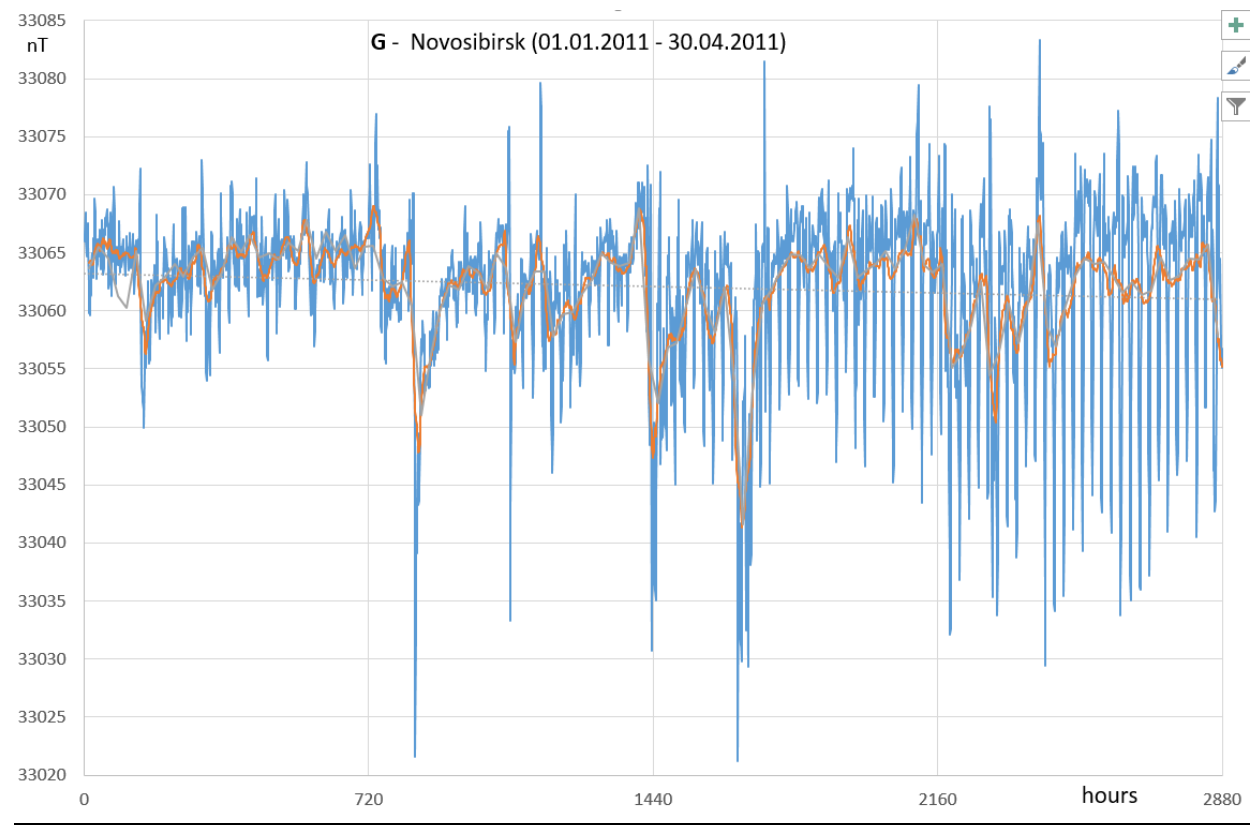

Fig 2. Average hourly and moving average daily values of local magnetic constant at the Novosibirsk Magnetic Observatory in January-March 2011.

It is easy to notice that the amplitudes of variations of average hourly values in spring (March and April) are significantly higher than in winter (January and February). This is due to the weak influence of the winter Siberian Sun on the daily course of the G, expressed in the lowering of its value in the local noon. But even in winter, the amplitude of these changes is sufficient to make it difficult to highlight events that are not related to 
the daily course. The comparison of the curves in the figure 3 for March 06-16.03.2011 clearly shows the advantage of using hourly daily mediation. This sliding 24-hour environment allows not only to highlight unique events free of daily variations, but also to consider their synchronization with start of earthquake preparation process with accuracy to one hour. On the curve of the moving daily average for March 2011 it can see in more detail features characterized by a significant (by 20-30 nT) decrease of local magnetic constant.

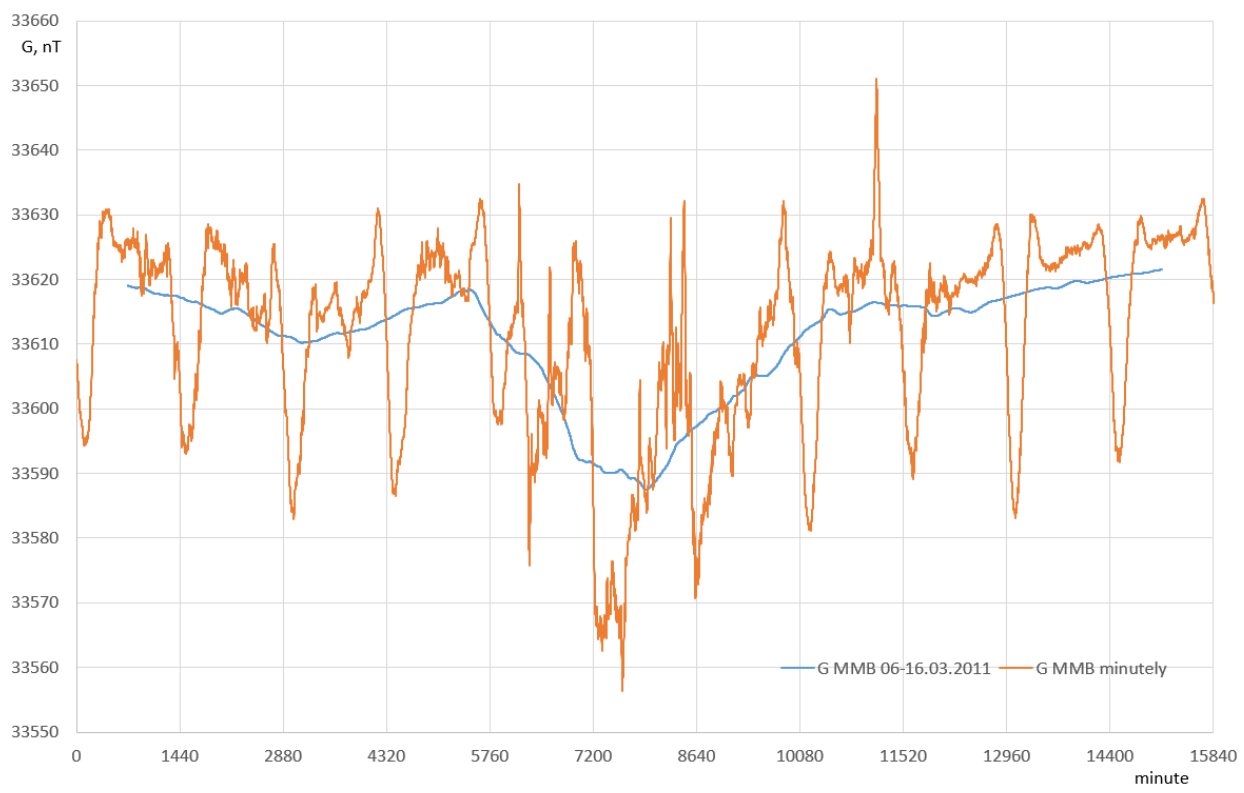

Fig. 3. Average hourly and moving average daily values of local magnetic constant at the Novosibirsk Magnetic Observatory for 06-16.03.2011.

The second event coincides with the Great Earthquake of Eastern Japan (March 11, 2011). In this case, the 24-hour $\mathrm{G}$ moving rate recorded for about 40 hours.

In all magnetic observatories located at a distance of thousands of kilometers from the epicenter of the earthquake on March 11, 2011, there was an increase in the rate of change in the magnitude of $\mathrm{G}$ in the coming to this date day. The use of a sliding daily average for each of the observatories allows you to receive graphs similar to the one presented in Figure 3 for the Novosibirsk Magnetic Observatory. Comparison of such curves allows us to raise the question of the beginning of preparation of an unusual geophysical event, not related to the daily rotation of the Earth, more confidently.

\section{Angles of the magnetic field during the Great Japanese Earthquake}

No less distinctly than in the power characteristic, the two above-mentioned features of magnetic field behavior in March 2011 are shown in the diagrams of the moving daily mean for declination and inclination, presented in figures 4 and 5. 


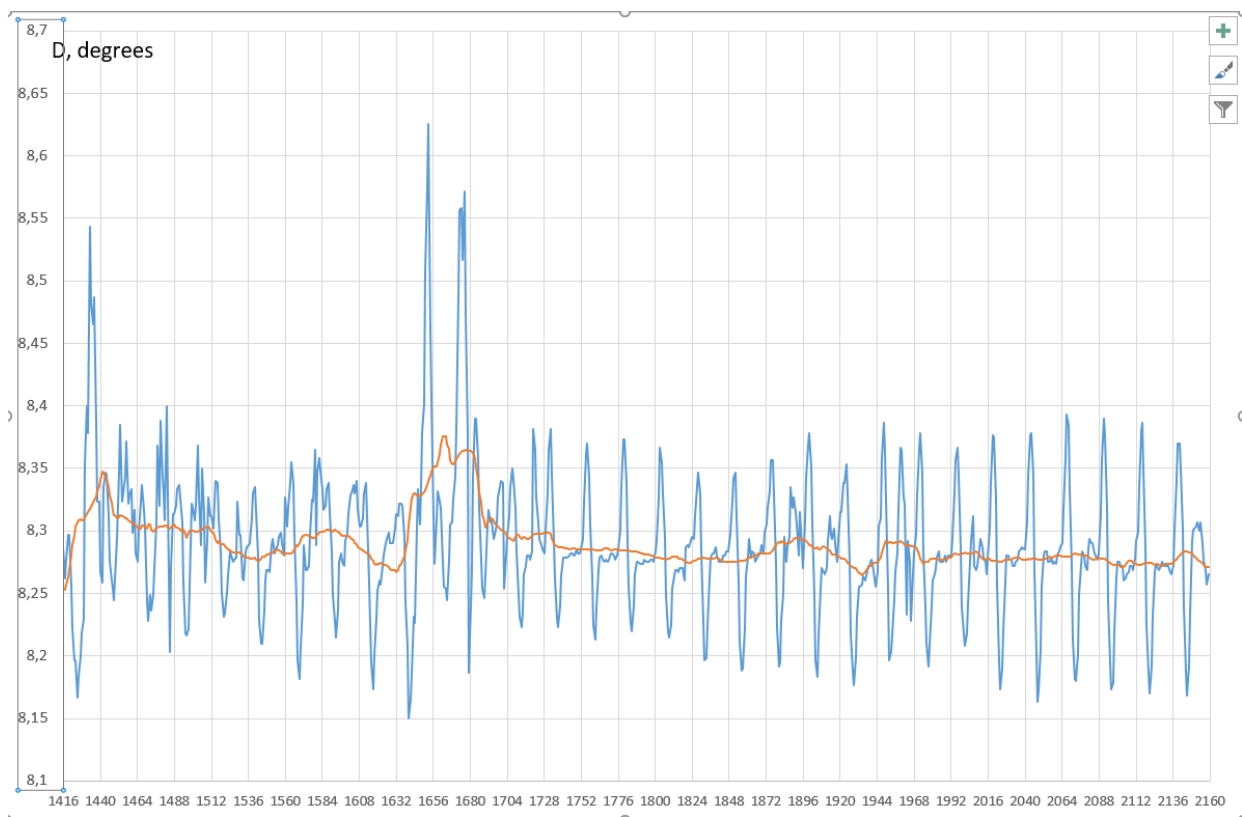

Fig 4. Average hourly and sliding 24-hour magnetic declination in Novosibirsk (March 2011).

The charts of average hourly values (Figure 4 and Figure 5) clearly show the daily variations of declination and inclination caused by the day-to-day rotation of the Earth. The calm course of the daily variation is disturbed during magnetic storms associated with the processes on the Sun.

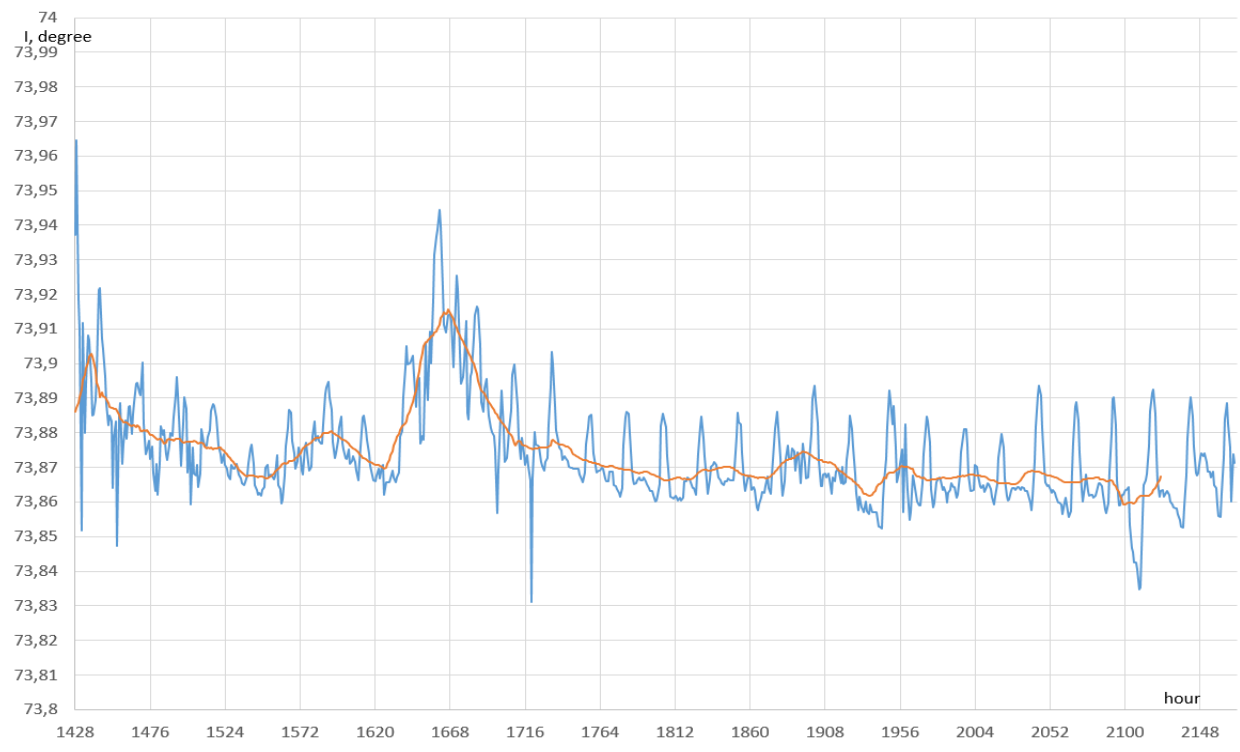

Fig 5. Average hourly and sliding 24-hour magnetic inclination in Novosibirsk (March 2011). 
For comparison with the more magnetically calm winter months, figures 6 and 7 on the same scale show similar patterns of declination and inclination for January 2011.

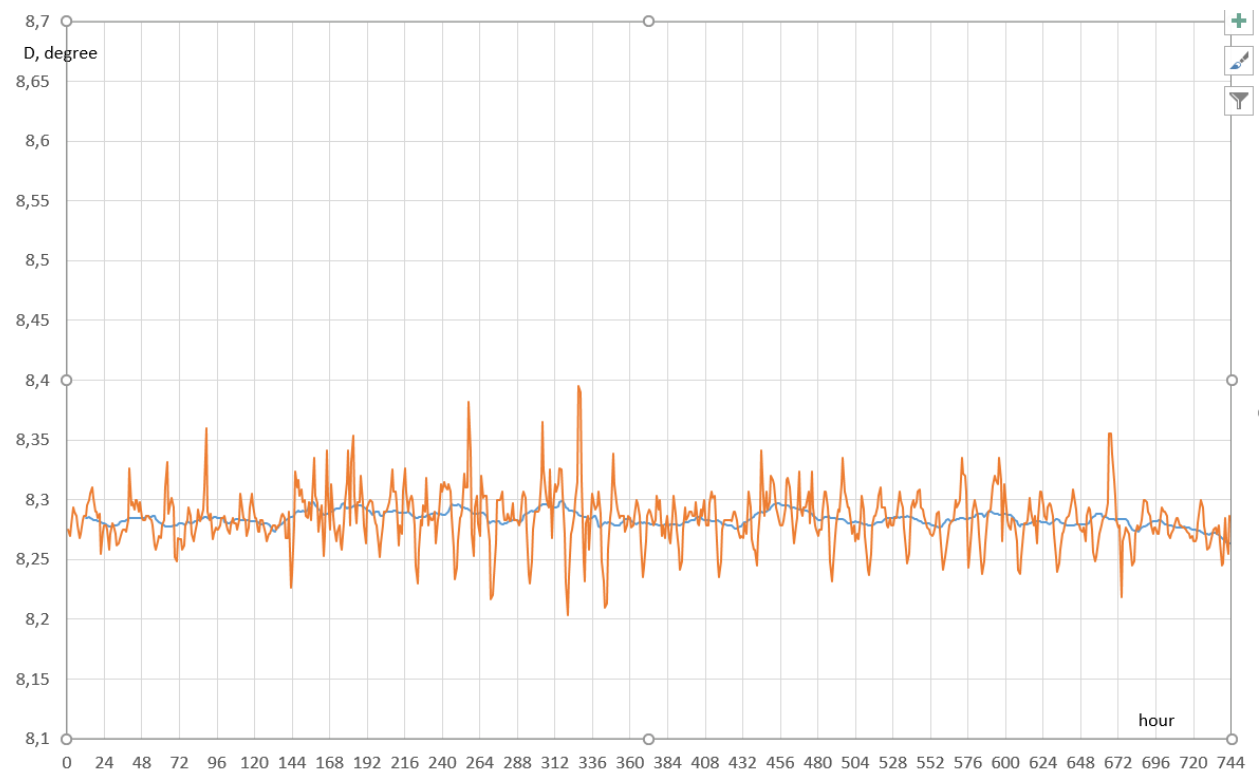

Fig 6. Average hourly and sliding 24-hour magnetic declination in Novosibirsk (January 2011)

As with the local magnetic constant, the amplitude of the daily course of angular elements of terrestrial magnetism (inclination and inclination) in January is significantly less than in March. It is a normal situation for the northern hemisphere.

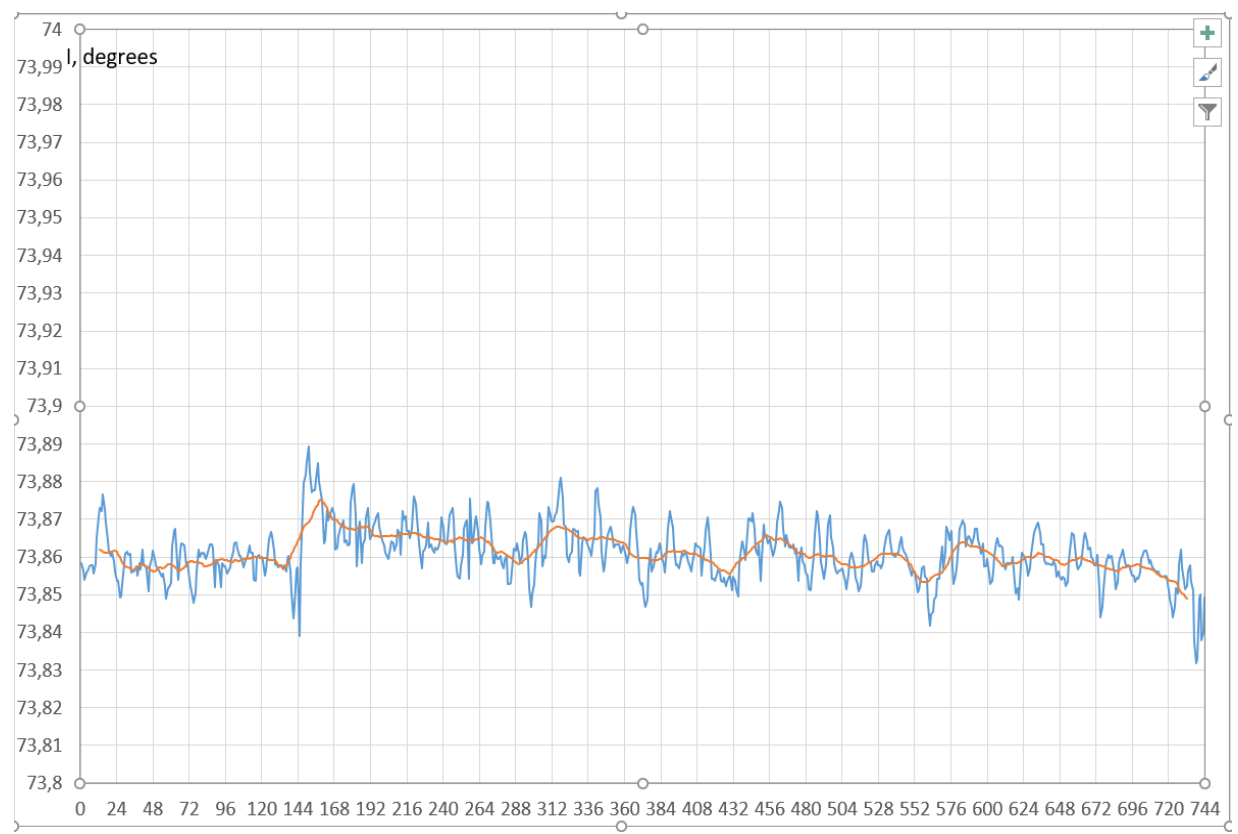

Fig 7. Average hourly and sliding 24-hour magnetic inclination in Novosibirsk (January 2011). 
However, even more clearly the change in the angular characteristics of the magnetic field can traced by the change in the trajectories of the virtual magnetic poles. Each of these trajectories is an integral characteristic of the movement of the magnetic pole, as opposed to angular elements (declination and inclination). For example, when the magnetic pole moves in the arc of a large circle in the direction of the magnetic observatory, the magnetic declination will not change. Similarly, there will be a constant magnetic inclination if the virtual magnetic pole moves across the surface of the globe, remaining at the same distance in the arc of a large circle from the observation point.

The second advantage of using the coordinates of virtual poles as a magnetic characteristic is the ability to compare patterns in the movement of these poles according to the data of different observatories. Figure 8 shows diagrams of the average daily speeds of the magnetic poles for a number of magnetic observatories [1].

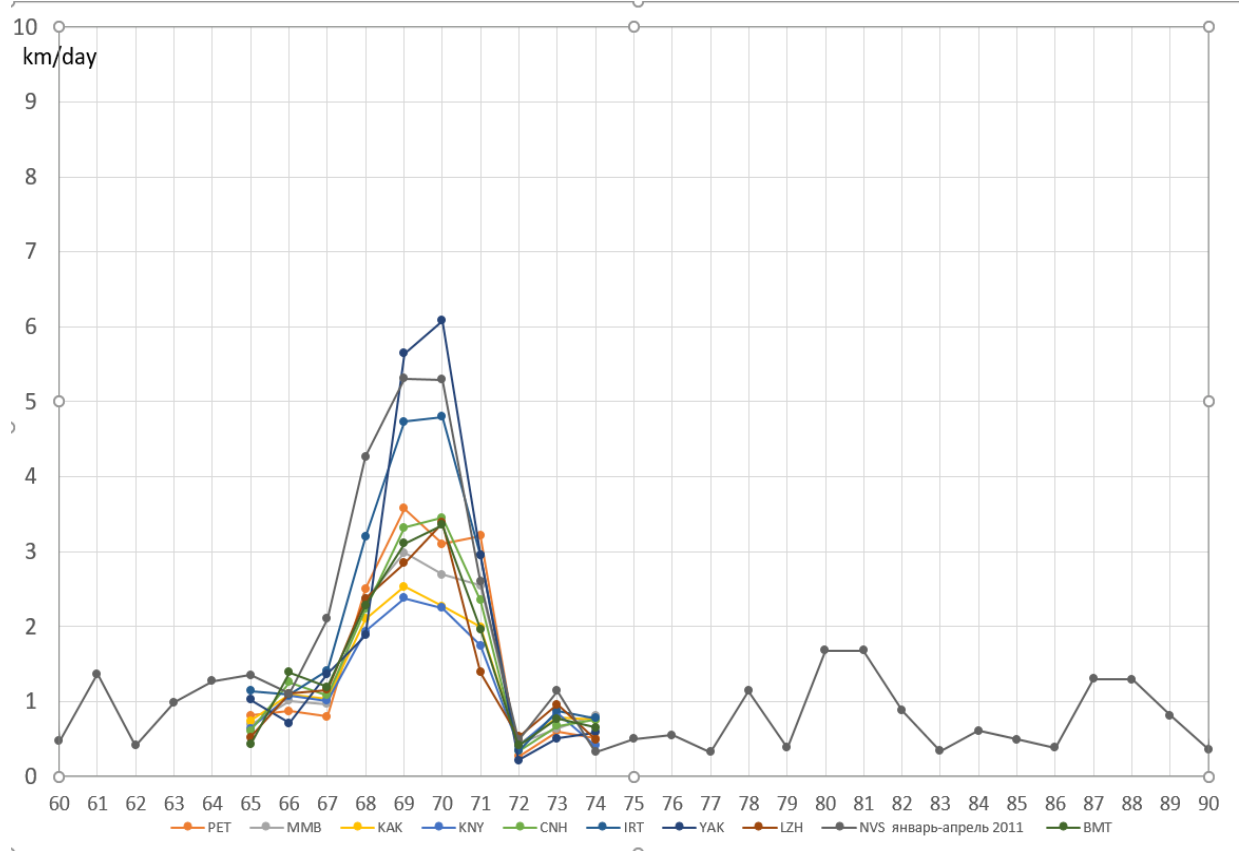

Fig 8 . Average daily speed of virtual magnetic poles for magnetic observatories.

You may notice that for all observatories, these speeds are significantly higher in days close to an earthquake.

The figure 9 shows two trajectories. The large curve in the area shows the movement of the virtual magnetic pole for 11.03.2011 according to minute data. And the second curve, smaller in size, shows the average daily position of the magnetic pole between March 6 and 16, 2011 according to the Novosibirsk Magnetic Observatory. First, the significant (about $5 \mathrm{~km}$ arc of a large circle) deviation of the magnetic pole position from the average for the specified days is noteworthy. In secondly, it noted, that the coordinates of the average daily position of the magnetic pole for March 11, 2011 do not coincide with any of the 1440 minutes coordinates. This situation is typical for a calm magnetic 
day, when the magnetic pole describes a figure similar to an ellipse in an area several times smaller than on indignant days (which includes the day of 11.03.2011).

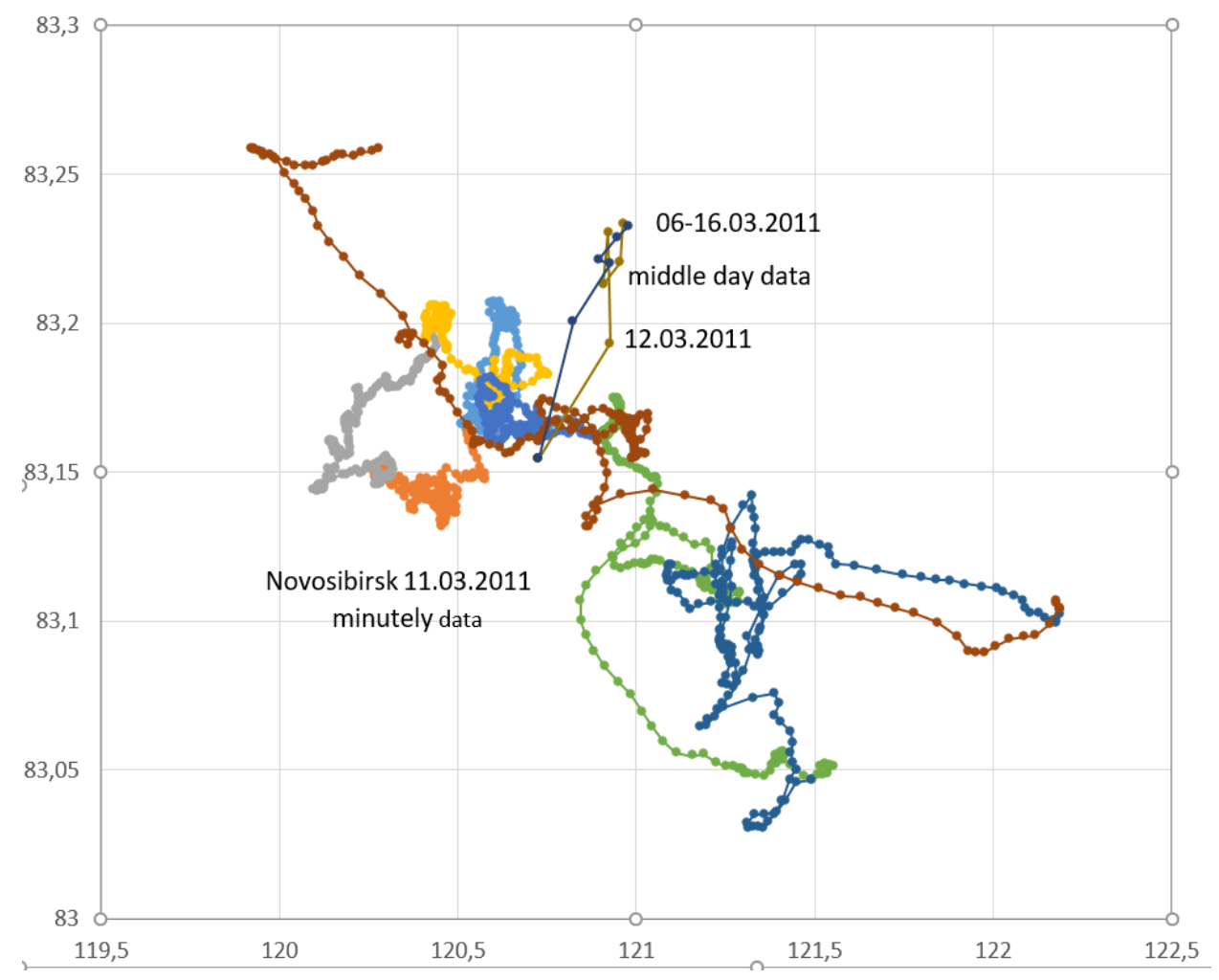

Fig 9. The movement of the magnetic pole on March 11, 2011 (by minute data) and from March 6 to March 16, 2011 (by average daily data) for the Observatory Novosibirsk.

Figures 10, 11 and 12 shows the position of the virtual magnetic pole, calculated on the average daily data of the same day (March 11) in different years in the magnetic observatories of Novosibirsk, Canoe and Paratunka. 


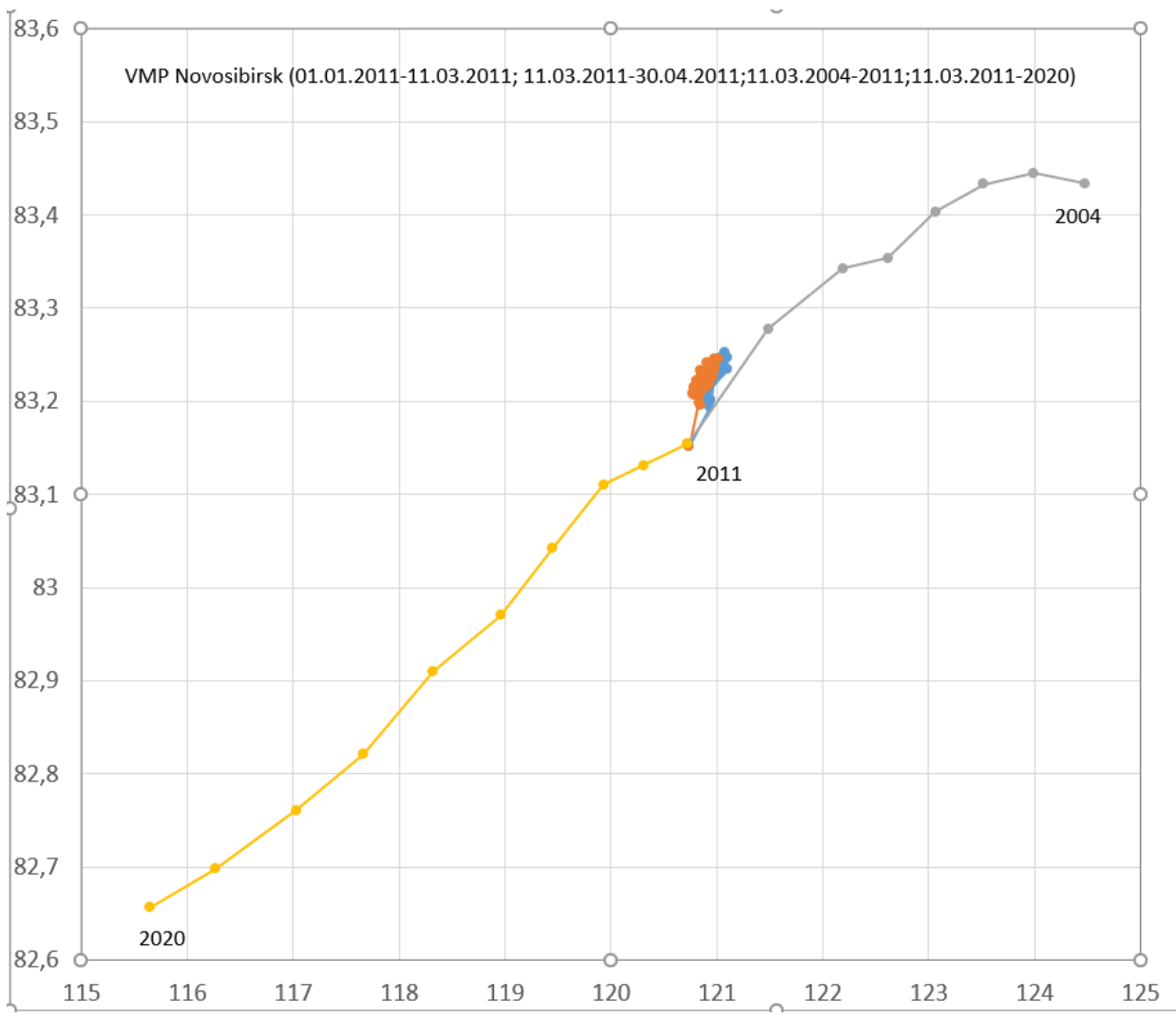

Fig 10. The movement of the magnetic pole from March 6 to 16, 2011 against the background of the curve of its annual movement according to the Novosibirsk Observatory (NVS).

The position of the magnetic pole in five-day intervals before and after March 11, 2011 on the figures 10-12 marked.

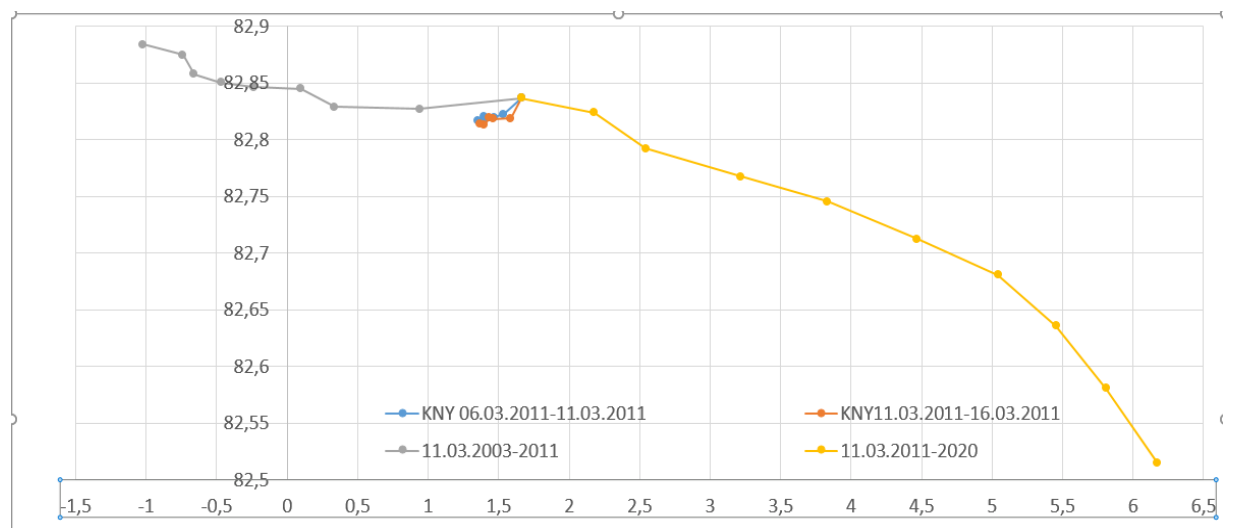

Fig 11. The movement of the magnetic pole from 6 to 16 March 2011 against the background of the curve of its annual movement according to the Kanoya Observatory (KNY). 
The general regularity observed in all Siberian, Japanese and Chinese magnetic observatories is "consistency" in the direction of the "emission" in the WMP movement, which takes place on March 11, 2011, with the general direction of the "century-old" movement of the UMP.

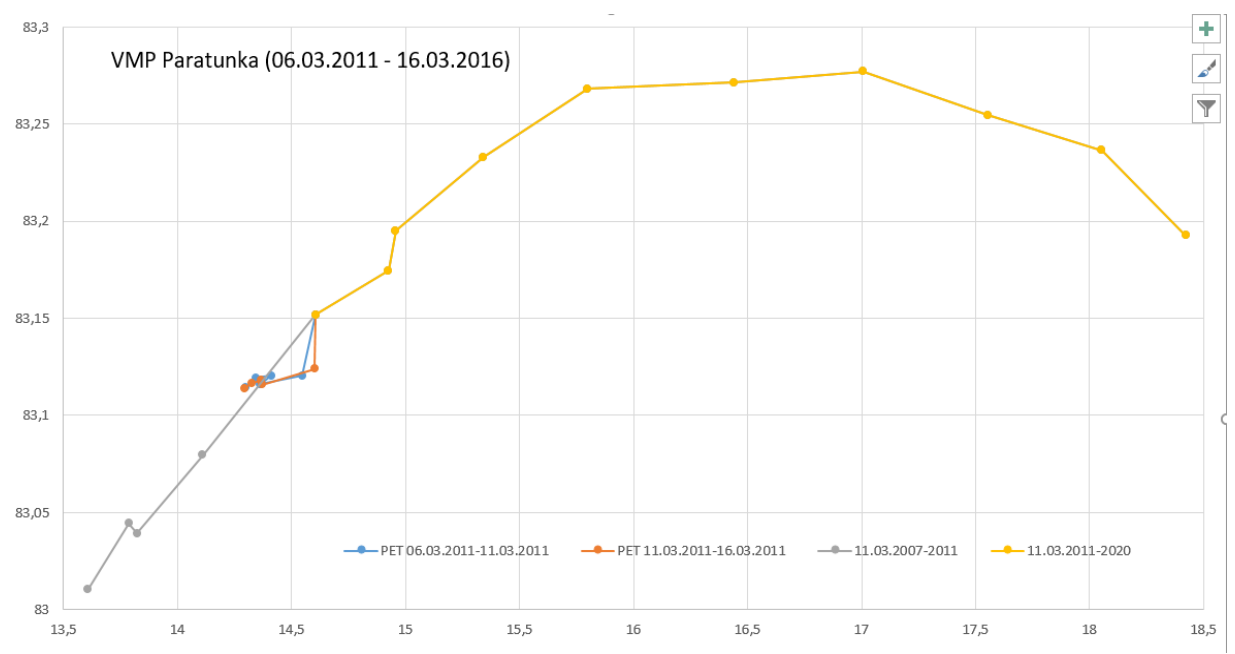

Fig 12. The movement of the magnetic pole from 6 to 16 March 2011 against the background of the curve of its annual movement according to the Paratunka Observatory (PET).

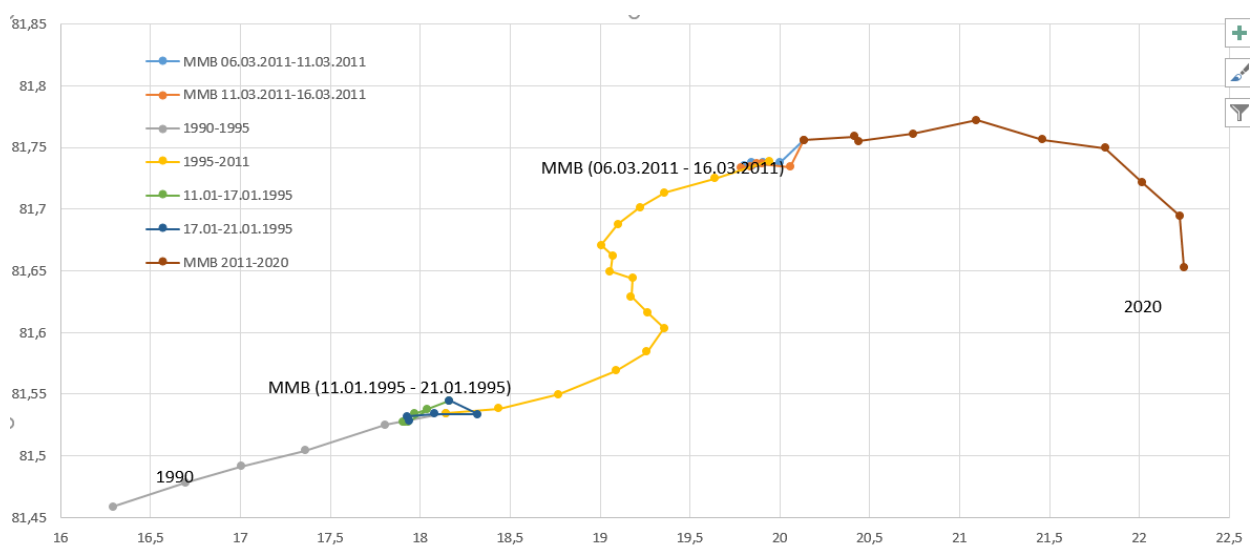

Fig 13. The movement of the magnetic pole from 6 to 16 March 2011 and from 11 to 21 January 1995 against the background of the curve of its annual movement according to the Memambetsu Observatory (MMB).

For more detailed identification of the features in the movement of magnetic poles, not associated with the daily rotation of the Earth, it is necessary, as in the case of the local magnetic constant, to calculate the moving average daily coordinates of the UMP in a step of at least one hour. Note that the data of modern magnetic observatories allow in real time to track the change in moving average daily positions of the virtual magnetic 
pole and the magnitude of the local magnetic constant in a minute-by-minute and even every second format.

It is possible that the analysis of "moving" average daily data on the movement of virtual magnetic poles and the change of local magnetic constant will allow to link with a high degree of confidence detected in the past and observed in the present features in the behavior of the geomagnetic field with the preparation of a major earthquake.

\section{Conclusions}

1. It is advisable to consider as possible geomagnetic harbingers of strong earthquakes integral power and angular characteristics of the Earth magnetic field: the local magnetic constant and coordinates of virtual magnetic poles.

2. Most informative and optimal in practical terms are the "moving" (in a step of 1 hour) average daily values of the above parameters.

3. The Great Earthquake of Eastern Japan, which occurred at 6 o'clock world time on March 11, 2011, was preceded by a decrease in for several tens of hours of moving average daily values of local magnetic constant in all the nearest magnetic observatories of Japan, China and Siberia.

4. The average daily speeds of virtual magnetic poles increase significantly a few tens of hours before a major earthquake and fall to their previous values in about the same time.

5. In order to monitor possible geomagnetic harbingers of large earthquakes, continuous monitoring of moving averages of local magnetic constant and realtime coordinates of virtual magnetic poles is necessary.

The authors thank all employees of magnetic observatories whose data used to calculate the coordinates of magnetic poles and value of the local magnetic constant.

\section{References}

1. INTERMAGNET (INTErnational Real MAGnetic NET). URL: $\mathrm{http} / / \mathrm{www}$.intermagnet.org/data-donnee/

2. L. A Bauer, Terr.Mag (Washington), V.19, 113-125 (1914)

3. N. N. Semakov, A. A. Kovalev, A. F. Pavlov, O. I. Fedotova, Science first hand, T.68, No.2, 96-107 (2016) 